\title{
CLASSIFICAÇÃO DOS TRATORES AGRÍCOLAS DE PNEUS EM FUNÇÃO DE SUA EFICIÊNCIA ENERGÉTICA ${ }^{1}$
}

\author{
Gastão Moraes da Silveira $^{2}$, Jacinto Gil Sierra ${ }^{3}$, Jaime Ortiz Cañavate ${ }^{4}$
}

\begin{abstract}
RESUMO
A redução nas reservas mundiais de petróleo contribui para o uso de máquinas com boa eficiência energética. A perda de energia nos tratores agrícolas de pneus ocorre, principalmente, no motor e na transmissão. Neste trabalho, são mostrados alguns índices, que representam a relação entre o consumo de combustível e a potência dos tratores. Assim, estabeleceu-se uma classificação dependente da eficiência do combustível. Os índices foram obtidos, a partir dos ensaios oficiais realizados nas estações que seguem os códigos OCDE, levando-se em consideração a eficiência do motor e da transmissão. O objetivo final é a proposta de um método, que classifica os tratores agrícolas de acordo com um índice, que representa sua eficiência energética.
\end{abstract}

Palavras-chave: índice de consumo maquina agrícola.

\section{ABSTRACT \\ Classification of agricultural tractor according to their energy efficiency}

The reduction of world oil reserves requires the use of machines with good energy efficiency. The engine and the transmission system are considered as major sources of the energy loss in agricultural tractors. In this study, some indices, representing the relationship between fuel consumption and tractor's available power, are developed to classify tractors on the basis of fuel efficiency. The indices were obtained from official tests performed in government research stations which follow OECD test Codes, taking into account the efficiency of the motor and the transmission. The final objective of the study is to propose a method that can be used to classify agricultural tractors according to an index representing energy efficiency.

Keywords: consumption index, farm machinery.

\footnotetext{
Recebido para publicação em 21.01.2007

${ }^{1}$ Trabalho financiado com bolsa de Pós Doutoramento da Fundação de Amparo a Pesquisa do Estado de São Paulo . FAPESP.

${ }^{2}$ Pesquisador Cientifico VI, MS, Dr., Livre Docente, Centro APTA de Engenharia e Automação, IAC/SAA/SP Rod. D. Gabriel PBCouto km65, CP26 CEP 13201-970, Jundiaí, SP Fone (011) 4582-8155, Ramal 137. E mail:

silveira@iac.sp.gov.br.

${ }^{3}$ Prof. Titular, Universidade Politécnica de Madrid, Escola Técnica Superior de Engenheiros Agrônomos, Departamento de Engenharia Rural. e mail: jacinto.gil@upm.es.

${ }^{4}$ Prof. Catedrático, Universidade Politécnica de Madrid, Escola Técnica Superior de Engenheiros Agrônomos, Departamento de Engenharia Rural e mail: jaime.ortizcanavate@upm.es.
} 


\section{INTRODUÇÃO}

No mercado existem diversos modelos de tratores. No momento da compra, a escolha do agricultor baseia-se na potência, conforto, facilidade de manobra e manutenção do trator, além do preço. O conhecimento da eficiência energética do trator poderia ser mais um item a ser considerado em sua seleção.

Para conhecer a eficiência energética e comparar os diversos modelos, é necessário basear-se em ensaios normalizados, que sejam idênticos para todos os modelos. Os únicos ensaios realizados na maioria das estações mundiais são estabelecidos pelos códigos OCDE. Assim, a única possibilidade de comparar os tratores é a utilização dos ensaios OCDE.

Os valores relativos à potência e consumo de combustível, são obtidos na tomada de potencia do trator, por meio de um dinamômetro. No ensaio da barra de tração, o trator traciona uma carga numa pista de concreto.

Baseando nos ensaios na tomada de potência, pode-se obter o valor do consumo especifico (kg ou litro de combustível por kW de potência), em diversos pontos do funcionamento do motor. Estes pontos, onde se fazem os ensaios no dinamômetro, são os mesmos para todos os tratores, e referem-se ao percentual da potência e rotação do motor em relação à potência nominal.

No ensaio na barra de tração, determinam-se a força de tração e o consumo de combustível do trator, alternando as diversas marchas na caixa de câmbio, ao mesmo tempo em que se traciona uma carga, obtendo-se, assim, dados de velocidade em diversas rotações. Quando o motor gira em determinada rotação, dividindo - se os valores obtidos de potência na barra de tração com aqueles da potência na tomada de potência, obtêm - se os diversos rendimentos da transmissão, e a média entre eles.

Com estas informações, obtêm-se índices que representam a eficiência energética dos tratores. O primeiro índice é a média ponderada do consumo especifico entre alguns pontos de potencia obtidos no dinamômetro, sendo denominado $\mathrm{Cj}$. Este índice somente representa a eficiência do motor. Dividindo-se este índice pelo rendimento médio da transmissão, obtém-se um segundo índice, que representa a eficiência do motor e da transmissão, denominado Cjt.

De acordo com Siemens e Bowers (1999), os custos de combustível e lubrificante representam, no mínimo, $16 \%$ chegando a atingir $45 \%$ dos custos totais das máquinas agrícolas, dependendo do tipo de combustível e do número de horas trabalhadas.

Segundo Zoz e Grisso (2003), o principal ponto a ser observado nos tratores agrícolas é o desempenho na barra de tração. A potência na barra de tração é definida pelo produto: da força multiplicada pela velocidade de deslocamento.

A American Society of Agricultural and Biological Engineers (2005) estabeleceu uma estimativa do consumo médio anual dos tratores acionados por motor diesel, de acordo com a seguinte fórmula: Consumo $(\mathrm{L} / \mathrm{h})=0,223 \times$ Potencia máxima na tomada $(\mathrm{kW})$.

Grisso et al. (2004) desenvolveram uma fórmula que permite a previsão do consumo de combustível de um trator $\mathrm{Lh}^{-1}$, para qualquer velocidade do motor em qualquer carga exigida, introduzindo, como variáveis independentes, a redução na velocidade do motor em relação à nominal, assim como a potência desenvolvida também em relação à nominal.

$$
\begin{aligned}
& C(L / h)=(0,22 X+0,096) \\
& {[1-(-0,0045 X . R V+0,00877 R V)] N_{n}}
\end{aligned}
$$

\section{Sendo:}

$X$ = quociente entre a potência fornecida pelo motor em cada ponto e a potencia nominal (decimais);

$\mathrm{RV}=$ redução da velocidade do motor em qualquer condição, em que o acelerador não esta no maximo em relação à que teria com - acelerador ao máximo, fornecendo a mesma potência (\%).

$\mathrm{Nn}=$ potencia nominal do trator $(\mathrm{kW})$ medida através da tomada de potência. 
Ressica et al. (1996) determinaram diferentes valores de consumo de combustível para o mesmo tipo de trabalho, em marchas distintas, orientando os usuários a reduzirem o consumo.

Um método para obtenção do desempenho de um motor, baseado nos dados de ensaios OCDE, foi desenvolvido por Harris (1992).

Segundo Golverk (1995), os motores diesel consomem menos combustível a cargas constantes, em comparação com cargas variáveis.

As condições de trabalho em pista de concreto foram estudadas por Degrell e Feuertein (2005), Os autores monitoraram um trator, medindo os valores instantâneos de velocidade de deslocamento, força de tração, rotação da tomada de potência, torque na tomada de potência, pressão e vazão dos sistemas hidráulicos.

Os objetivos do presente trabalho são: identificar os fatores, que intervêm na eficiência energética dos tratores agrícolas; analisar as relações entre os parâmetros do trator e a eficiência energética; desenvolver índices relacionados à eficiência energética dos tratores, de acordo com dados de ensaios normalizados; fornecer aos agricultores uma informação simples, útil e prática sob o ponto de vista energético do que eles vão comprar. É proposta, também, uma classificação de alguns tratores agrícolas comercializados no Brasil de acordo com a eficiência energética.

\section{MATERIAL E METODOS}

Os dados do presente trabalho foram obtidos na Estação de Mecânica Agrícola de Madrid, pertencente ao Ministério da Agricultura da Espanha. Foram estudados os dados de 37 modelos de tratores ensaiados, nos seguintes Centros de Ensaio: Alemanha (DLG), Inglaterra (Silsoe), Estados Unidos (Nebraska), França (Cemagref), Espanha, Polônia, Turquia e Finlândia, sendo que os relatórios publicados por estes centros, seguem o Código Normalizado OCDE para ensaio de tratores.

$\mathrm{O}$ índice $\mathrm{Cj}$ reflete-se à eficiência energética do motor do trator, medida mediante a da tomada de potência. Para calcular $\mathrm{Cj}$, utiliza-se o consumo especifico médio dos pontos assimilados na Figura 1. O procedimento é o seguinte: multiplica-se o consumo dos pontos $8,9,6,7$ e 10 por 2 , considerando-se que estes possuem uma freqüência de funcionamento maior que os outros cinco pontos ( 1 a 5 ) que aparecem na descendência da curva apresentada na respectiva Figura.

Segundo Sierra et al. (2006) para obtenção dos consumos específicos nos pontos 1, 2, até 10, (Figura 1) se aplica - se a equação de Grisso et al.(2004).

$O$ índice tem a seguinte expressão matemática:

$$
\mathrm{C}_{\mathrm{j}}=\frac{\begin{array}{cc}
\sum \mathrm{C}_{\text {esp } \mathrm{i}}+2 & 10 \\
\sum \mathrm{C}_{\text {esp } \mathrm{i}} & \mathrm{i}=6
\end{array}}{}
$$

\section{5}

Assim, obtém-se o consumo especifico médio em L.kWh ${ }^{-1}$, baseado nos pontos mais freqüentes de utilização sendo perfeitamente valido para realizar as estimativas comparativas da eficiência energética.

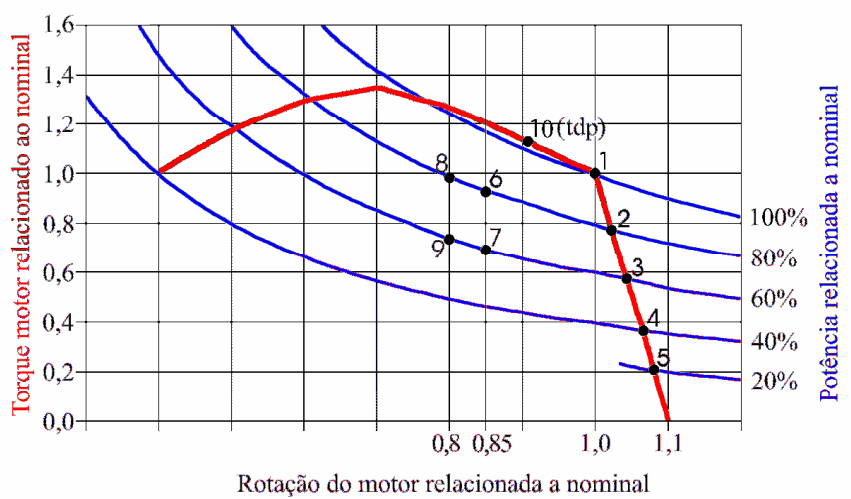

Figura 1. Pontos cujo consumo especifico é levado em consideração, para calcular o valor do índice relativo à eficiência do motor. 
Quadro 1. Distribuição das horas de funcionamento anual do parque de tratores na Espanha, de acordo com a utilização da potência do motor

\begin{tabular}{|c|c|c|c|}
\hline \multirow{2}{*}{$\begin{array}{c}\text { Potencia } \\
\text { CV }\end{array}$} & \multicolumn{3}{|c|}{$\begin{array}{c}\text { Distribuição do numero de horas trabalhadas por tipo } \\
\text { de trabalho } \\
(\%)\end{array}$} \\
\cline { 2 - 4 } & $\begin{array}{c}\text { Tomada de } \\
\text { potencia }\end{array}$ & $\begin{array}{c}\text { Tração }<8 \\
\text { km/h }\end{array}$ & $\begin{array}{c}\text { Tração }>8 \\
\text { km/h }\end{array}$ \\
\hline$<30$ & 37,14 & 29,43 & 32,82 \\
\hline $30-49$ & 34,97 & 34,61 & 30,42 \\
\hline $50-69$ & 35,26 & 34,16 & 30,58 \\
\hline $70-89$ & 31,70 & 37,23 & 31,07 \\
\hline $90-109$ & 25,68 & 42,92 & 31,40 \\
\hline $110-129$ & 27,50 & 43,14 & 29,36 \\
\hline $130-150$ & 19,48 & 46,94 & 33,58 \\
\hline$>150$ & 12,43 & 57,47 & 30,10 \\
\hline
\end{tabular}

Em tratores, a energia produzida no motor é transmitida para as rodas, por meio da caixa de cambio, diferencial, redutores finais e outros elementos. Em todos esses dispositivos ocorrem perdas. O ensaio em que a transmissão atua e, portanto, a avaliação de sua eficiência poder ser realizada, é o ensaio na barra de tração. As perdas de energia são produzidas durante a transmissão e no contacto roda-solo. No contacto roda-solo ocorre perdas devidas ao rolamento e deslizamento. As perdas, por rolamento não são avaliadas no ensaio segundo o código OCDE, mas o deslizamento é. Este dado figura nos boletins de ensaio, junto à força de tração, velocidade de deslocamento, potência rotação do motor e consumo.

As estatísticas de distribuição dos tempos de trabalho podem agrupar, tarefas, como adubação, tratamento fitossanitário, e colheita, bem como aquelas de tração em velocidades lentas (inferiores a $8 \mathrm{~km} / \mathrm{h}$ ) e as de trabalho mais rápidas, originando os dados do Quadro 1.

O valor do índice $\mathrm{Cj}$ obtido para cada trator deve ser modificado, levando-se em conta a influência da transmissão. Para realizar esta modificação, de acordo com a informação apresentada no Quadro 1, deve- se dividir o valor do índice $\mathrm{Cj}$ de cada modelo em três partes proporcionais ao tempo, que o trator dedica a cada tipo de trabalho. Assim, uma onde predominam trabalhos lentos, e outra em trabalhos rápidos, dividida pelo valor médio dos coeficientes $\eta$, calculados para tais velocidades.

As atividades de trabalho dos tratores, foram agrupadas em três categorias: tarefas onde se utiliza a tomada de potencia; trabalhos na barra de tração com velocidades menores que $8 \mathrm{~km} / \mathrm{h}$; e trabalhos na barra de tração em velocidades superiores a $8 \mathrm{~km} / \mathrm{h}$. O valor do índice Cj é dividido em partes proporcionais à percentagem do tempo durante o qual trator é usado na tomada de potência, barra de tração em velocidades menores que $8 \mathrm{~km} / \mathrm{h}$, e em velocidades superiores a $8 \mathrm{~km} / \mathrm{h}$.

$$
\begin{aligned}
& C_{j} \text { tdp }=C_{j} \times \frac{\% \text { do tempo trabalho na tdp }}{100} \\
& C_{j}<8 \mathrm{~km} / \mathrm{h}=C_{j} \times \frac{\% \text { tempo trabalho }<8 \mathrm{~km} / \mathrm{h}}{100} \\
& C_{j}>8 \mathrm{~km} / \mathrm{h}=C_{j} \times \frac{\% \text { tempo trabalho }>8 \mathrm{~km} / \mathrm{h}}{100}
\end{aligned}
$$


O novo índice da eficiência da transmissão é:

$C_{j t}=C_{j} t d p+\frac{C_{j}<8 \mathrm{~km} / \mathrm{h}}{\eta<8 \mathrm{~km} / \mathrm{h}}+\frac{C_{j}>8 \mathrm{~km} / \mathrm{h}}{\eta>8 \mathrm{~km} / \mathrm{h}}$

Sendo $\eta<8 \mathrm{~km} / \mathrm{h}$ e $\eta>8 \mathrm{~km} / \mathrm{h}$, a média dos valores dos coeficientes $\eta$, medidos em cada trator abaixo e acima de $8 \mathrm{~km} / \mathrm{h}$, baseia-se na eficiência do motor e da transmissão. Deste modo, pode-se usar Cjt como índice para a classificação de tratores, de acordo com sua eficiência energética.

Por exemplo, para os modelos de tratores cuja potência varia entre 70 e $89 \mathrm{cv}$, o novo índice da eficiência de transmissão obedece a formula:

$C_{j t}=31,7^{*} C_{j}+\frac{37,23^{*} C_{j}}{\eta \text { médio } a<8 \mathrm{~km} / \mathrm{h}}+\frac{31,07^{*} C_{j}}{\eta \text { médio } a>8 \mathrm{~km} / \mathrm{h}}$

É preferível não desenvolver a classificação da eficiência energética comparando unicamente o valor do índice Cjt dos tratores, a classificação deve também considerar o valor $\mathrm{Cjt}$ relacionado à potencia.

\section{RESULTADOS E DISCUSSÃO}

Foi definido um limite contínuo, fixado em função do valor da potência nominal. Para isto, utilizando a Estatística Experimental e o programa Microsoft Ofice Excel, determinouse a curva de regressão, que estabeleceria de maneira mais exata possível a relação entre Cjt e a potência nominal. Os resultados obtidos são apresentados na Figura 2.

A Figura 3 mostra uma nuvem de pontos de coordenadas Potência nominal/ índice Cjt. A linha que melhor representa a tendência dessa nuvem de pontos é a exponencial, cuja equação é

$\mathrm{C}_{\mathrm{jt}}=0,3832 \mathrm{e}^{-0,0002 \mathrm{Nn}}$

Sendo $\mathrm{Nn}$ a potência nominal

Considerando as diferenças entre os diversos valores de Cjt e com o objetivo de que a classificação da eficiência energética divida os tratores em 5 grupos, valor que parece razoável, optou-se por intervalos de 6 em $6 \%$ do consumo médio expresso pela linha de regressão, para cima e abaixo da linha média.

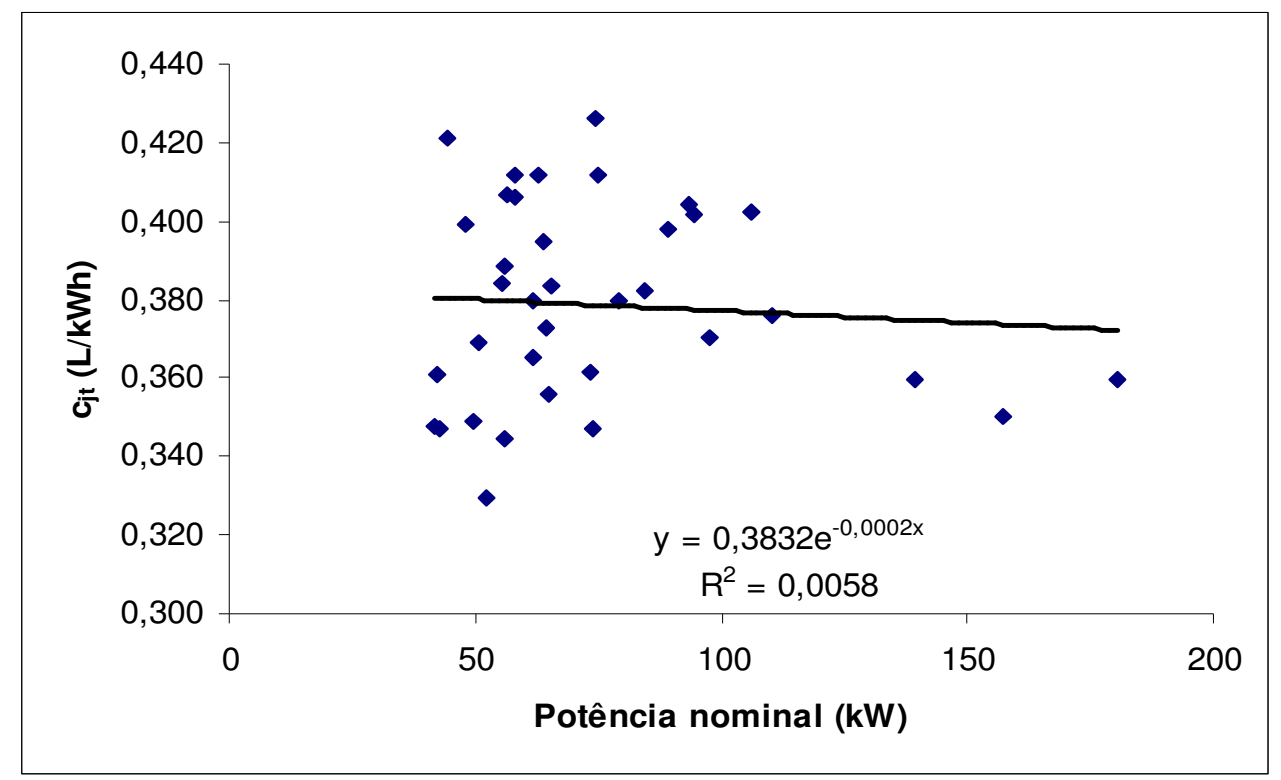

Figura 2. Coordenadas dos valores Potência Nominal - índice Cjt para os modelos de tratores estudados. 
A Figura 3 está dividida em 5 faixas. A faixa central tem, como limite superior, o valor médio Cjt expresso pela Equação 8, aumentado em 3,0\% e, como limite inferior, o valor médio Cjt expresso pela formula 1, reduzido em $3,0 \%$. A faixa imediatamente superior chega até 0 valor médio incrementado em 9,0\%, sendo a mais alta a partir deste limite. A faixa intermediaria inferior à central tem, como limite inferior, o valor médio reduzido em $9,0 \%$, e a mais baixa deste limite em diante.

Segundo esta metodologia, os tratores se classificam em função do valor Cjt dentro do intervalo de potência nominal correspondente, conforme Figura 3. Os pontos desta figura referem-se a tratores que foram, ou são, vendidos no mercado brasileiro.

Baseado nesta metodologia, os tratores mais eficientes estão classificados na categoria $A$, os menos eficientes na $E$ e os demais em faixas intermediárias de acordo com o seu Cjt e potência nominal. (Figura 4).

A potência dos tratores, classificados na categoria $A$, varia de 52 a $56 \mathrm{~kW}$. Os da categoria $\mathrm{B}$, de 41 a $158 \mathrm{~kW}$; na $\mathrm{C}$, de 50 a $180 \mathrm{~kW}$; na D, 47 a $120 \mathrm{~kW}$; e na E, de 44 a 76 $\mathrm{kW}$.

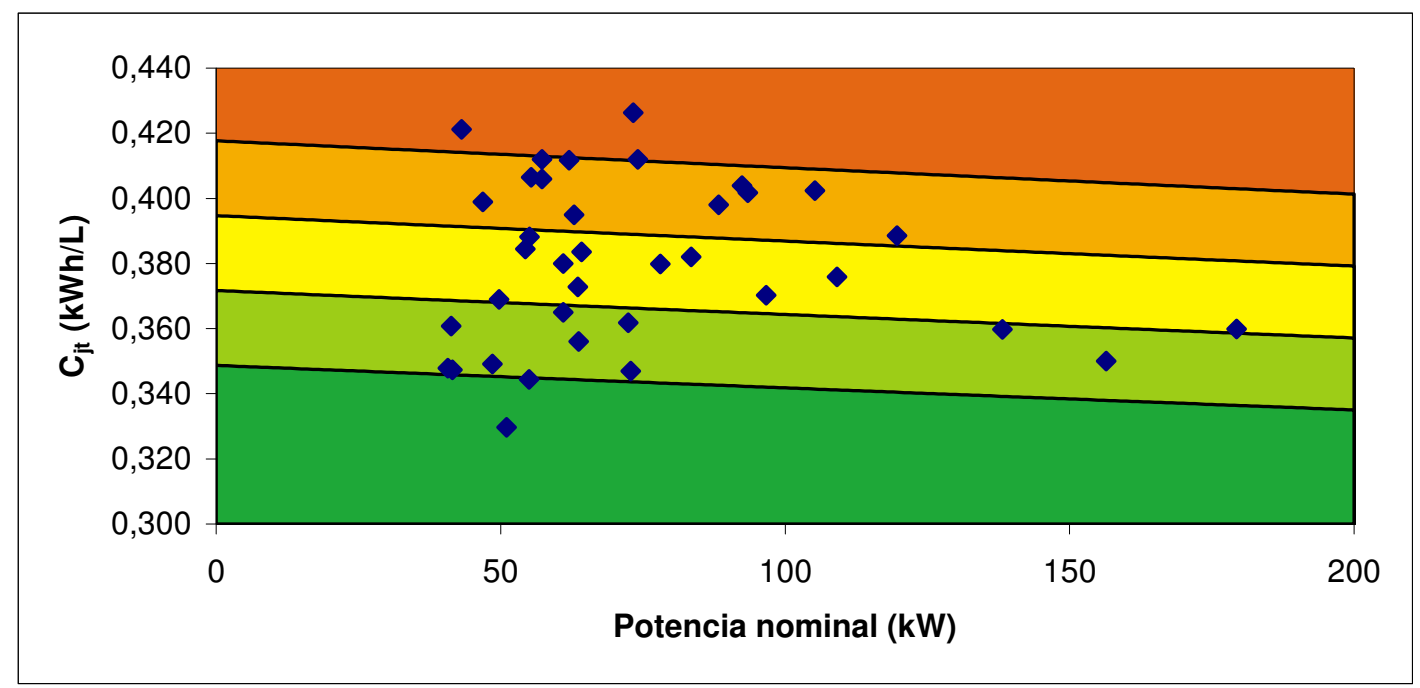

Figura 3. Classificação energética em função do Cjt e da potência nominal.

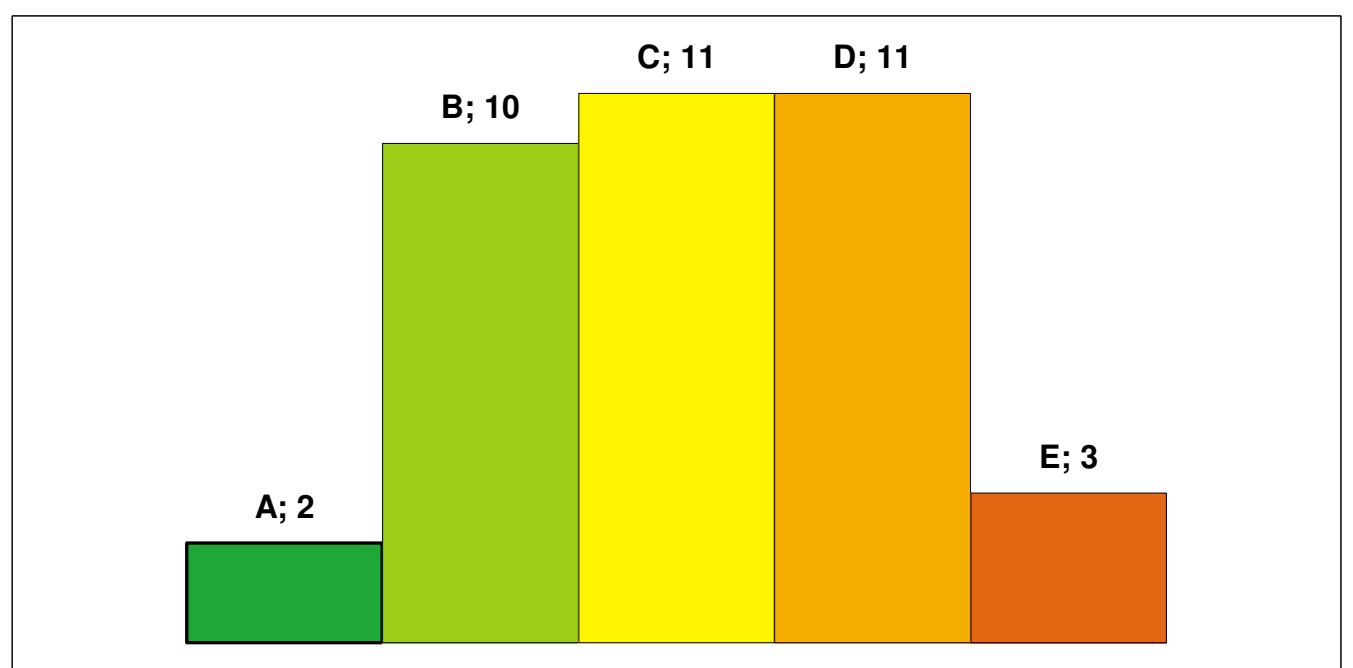

Figura 4. Distribuição dos modelos estudados por categoria em função de sua eficiência energética 


\section{CONCLUSÕES}

- Dois tratores estão classificados na categoria A, como sendo os mais eficientes e três na categoria $E$, como os menos eficientes. Existem também 10 na categoria $B, 11$ na categoria $C$ e 11 na categoria D. Portanto, poucos estão modelos nas categorias mais e menos eficientes, mas a maioria está em categorias intermediárias.

- Por estar baseada na linha média de regressão, que é inclinada, a classificação não permite fazer comparação entre os modelos dentro de cada faixa. Outro fator é a dificuldade em comparar os modelos em cada faixa, sendo as potências diferentes.

- Nestes intervalos, o número de modelos classificados em cada categoria segue uma distribuição normal, uma vez que existem poucos modelos na categoria mais eficiente $A$, também poucos na menos eficiente $E$ e muitos nas intermediarias $B, C$ e D.

\section{REFERÊNCIAS BIBLIOGRÁFICAS}

AMERICAN SOCIETY OF AGRICULTURAL AND BIOLOGICAL ENGINEERS (ASBE) Agricultural Machinery Management EP 496.2 Standards 2005. $52^{\mathrm{a}}$ edition. ASABE. St. Joseph (Michigan).

DEGRELL, O. FEUERSTEIN. DLG.PowerMix ein praxisorientierter Traktorentest "Testzyklen". Conference Agricultural Engineering VDI-Meg. Tagung Hannover 4-5 noviembre 2005.

GRISSO, R.D., KOCHER, M.F., VAUGHAN, D.H. Predicting tractor fuel consumption. Applied Engineering in Agriculture. St. Joseph, Mich. V 20, n.5 p.553-561, 2004.
GOLVERK, A.A. Diesel engine performance maps under variable loadings. Internal Conmustion Engine Division Spring Technical Conference. ASME. Marietta (OH), abril 1995.

HARRIS, H.D. Prediction of tractor performance using OCDE standard test data. Journal of Agricultural Engineering Research. v. 53, p. 181-193, 1992.

RESSICA, J.M., BOTTA, G.F., DE SIMONE, M.E. Consumo de combustible del tractor en relacion a las curves caracteristicas del motor y a su transmisión. Congresso Argentino de Ingenieria Rural. www.unlu.edu.ar/magagro/CADIR 96.pdf (disponible on line).Buenos Aires, 1996.

SIERRA, J.G; ORTIZ-CANÃVATE, J; GILQUIRÓS, V; CASANOVA,J. Energy efficiency in agricultural tractors. A methology for their classification. In: CIGR WORLD CONGRESS, 16, Bonn. Resumos...Bonn: CIGR, 2006. p. 111 112.

SIEMENS, J.C. , W.W. BOWERS. Machinery management: how to select machinery to fit the real needs of farm managers. Farm Business Manegement (FMB) series, John Deere Publishing, East Moline, II. 1999.

ZOZ, F. \& GRISSO, R.D. Traction and tractor performance. ASAE Distinguished lecture Series, 27. St. Joseph, Mich.: ASAE, 2003. 